\title{
THE PROBLEM OF TUBERCULOSIS IN CHILDHOOD
}

\author{
By A. Margaret Macpherson, M.D., F.R.C.P. \\ Physician, Elizabeth Garrett Anderson Hospital, London; Assistant Physician, Children's Contact Dept., \\ Brompton Hospital
}

Tuberculosis in children has to be considered from more points of view than has tuberculosis in adult life. Since congenital tuberculosis is of rare occurrence, virtually all children begin life free from tuberculous infection but during childhood an appreciable number become infected. The problem of infection by the tubercle bacillus in childhood may present itself from the epidemiological standpoint, from the aspect of case finding among the child's associates, and gradually merges into problems connected with tuberculous morbidity resulting from the primary infection.

How many children are likely to be infected at various ages, how seriously must this infection be taken; how should primary lesions be dealt with and how often may serious consequences be expected to arise ?

These are some of the questions which are frequently asked.

\section{Infection}

Although there is evidence to show that fewer children now become infected compared with 20 years ago, primary tuberculosis in childhood is still a problem of some magnitude. The most recent general survey of the incidence of tuberculous infection, as shown by tuberculin testing, indicates that between 40 to 57 per cent. of children at 15 years of age in England and Wales have had a primary infection. ${ }^{1}$

In the population as a whole the rate of infection varies in different age groups. Younger children whose horizon is limited are less likely to be infected than older children. Immediately after leaving school, the rate of infection, in urban districts, rises more sharply. The figures quoted above represent the condition present in 1949 and 1950. Improvements in the early diagnosis of active cases and in treatment of sputum-positive cases, together with the wider use of B.C.G. inoculation may result in considerable alteration of these rates in the not too distant future.

Since roughly half of the population of children in England and Wales have primary tuberculous lesions it is obvious that the majority of these 3 lesions give rise to no clinical symptoms. The problem then presents itself, how many of these. primary lesions are likely to cause trouble and $\stackrel{\omega}{A}$ which are the ones that are likely to do so?

\section{Incidence of Clinical Manifestations in Infected Children}

It is clear that the age at which primary tuber- $\vec{c}$ culous infection takes place is of great importance. Infection under 3 years of age must be regarded seriously; primary lesions at this age may Bece quickly followed by serious and not infrequen $4 y^{\oplus}$ fatal complications. Between the ages of 5 훙으 years, primary infection is less likely to give rise to clinical symptoms and signs. Tuberculosis, even in this age group is significant and although death rates at this age are at a lower level for all $\underset{Q}{\mathbb{Q}}$ diseases, tuberculosis is still high in proportion to other diseases. The findings of Hyge among schoolchildren in contact with a teacher with positive sputum show that tuberculous infectiono at this age cannot be treated lightly. He found that of the 70 children infected, $4 \mathrm{I}$ had pulmonary 3 . tuberculosis and of these 37 had tubercle bacillio recovered from gastric lavage. ${ }^{2}$

In later childhood and adolescence, it appearsi that primary infection may not infrequently be responsible for pulmonary lesions of the infiltrative type usually associated with young adult life. These lesions, though less dramatic in the im mediate post-primary stage, lead eventually ton chronic tuberculous lesions with long periods of invalidism and insecurity.

Other factors also no doubt have their place int the aetiology of tuberculous morbidity such aso extent of exposure to infection, environmento heredity and nutrition.

The incidence of morbidity due to tuberculous? infection in children is difficult to measure. The relation between the mortality and morbidity figures is altering and mortality figures are a less? 
useful guide than they used to be to the significance of tuberculous disease in childhood. This is specially so in the case of tuberculous meningitis. Before the use of streptomycin all established cases of meningitis had a fatal outcome, the rate of incidence and the mortality rate for this type of tuberculous lesion corresponding to each other; now the gap between them is widening, as no doubt it is for other forms of tuberculosis in young children.

A recent attempt to estimate morbidity figures in children infected before 5 years of age has been made in Newcastle by Cammock and Miller. They consider that of children under 5 years who have had primary tuberculous infection, 3 to 5 per cent. are liable to develop serious complications. Of the $25^{8}$ infected children in Newcastle, who were traced, it was found that I.5 per cent. developed bone lesions and 4 . I per cent. developed tuberculous meningitis or miliary tuberculosis within a minimum of two years from infection. ${ }^{3}$ Apart from these there are the children who suffer from general ill-health as a result of gross glandular infection or caseating tuberculous lesions in the lung and those who, although they eventually recover from the tuberculous lesions, are left with chronic pulmonary lesions resulting from bronchial occlusion.

In Rochester, New York, Beaven found in the $\circ$ to 14 years age group that there were 78.7 clinical cases of tuberculosis per 1,000 infected persons, a morbidity rate which was considerably higher than that in older age groups.

Looking at the problem from the point of view of the individual child, the first question is to establish whether or not the child is infected; if infected, to consider the likelihood of lesion to give rise to trouble. If the child is under 3 to 5 years, there is considerable risk of illness of greater or less severity: if the child is over 5 years and under ro years there is much less chance of developing clinical tuberculosis: if over to years, and more especially if $I 5$ to 17 years, the outcome will to a large extent depend on whether the primary infection is recent or not. It may be possible to establish this if previous tuberculin tests have been negative, or the history of recent exposure to infection may make recent infection likely. If this is so, then there is a risk that the recent lesion may spread rgiving rise to chronic infiltration. 6

\section{Diagnosis}

The classical pictures of acute tuberculous bronchopneumonia, pleurisy, miliary and meningeal tuberculosis, peritonitis and bone lesions need not be described here. These obvious lesions, apart from bronchopneumonia and pleurisy, are in reality complications of a primary lesion which in itself may be much less easily recognized.

A primary lesion may develop and resolve without its presence being suspected; it may cause ill-health which is not readily recognized as being due to tuberculous infection, or it may give rise to persisting lesions in the lungs, nontuberculous in themselves but resulting from the original primary lesion. From the primary complex, tubercle bacilli may enter the blood stream in small or large numbers, giving rise to the haematogenous lesions. It is considered by many observers that haematogenous infection is common although usually not manifest.

\section{$\mathrm{X}$-ray Findings}

Symptoms and clinical signs although they may be aids are usually too indefinite to be of any use in making a diagnosis of early primary tuberculosis. Radiography will provide the most useful evidence of a primary lesion. On the other hand the absence of any evidence in the radiogram does not necessarily exclude a primary lesion; the lesion may be too small to show, or at too early a stage to show, or may be hidden by the mediastinal or heart shadows. Evidence of a primary complex when

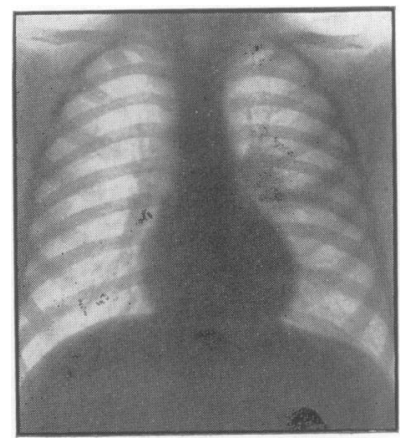

X-RAY I. - Primary tuberculosis with enlargement of glands at the left hilum.

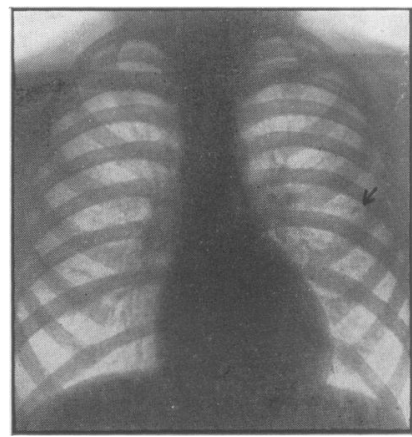

X-RAY 2. - Same child, three years later. showing calcification in the hilar glands and in the lung lesion.

(X-Ray I $\mathfrak{G}^{2}$ reproduced by courtesy of the Royal Society of 
visible is shown by the enlargement of glands at the hilum or supra-hilar region. The primary lesion in the lung may or may not be seen. It is often not possible to detect it until healing with calcifica tion develops and makes it more visible in the radiogram (X-ray $\mathrm{I}$ and 2 ). When the early lung lesion can be seen it is small and localized, some. times slightly flocculent and sometimes homogenous and demarcated from surrounding lung. There is, however, much variation in size of what appears to be the primary lesion.

The size of the enlarged glands also varies greatly. The glands tend to be larger in the

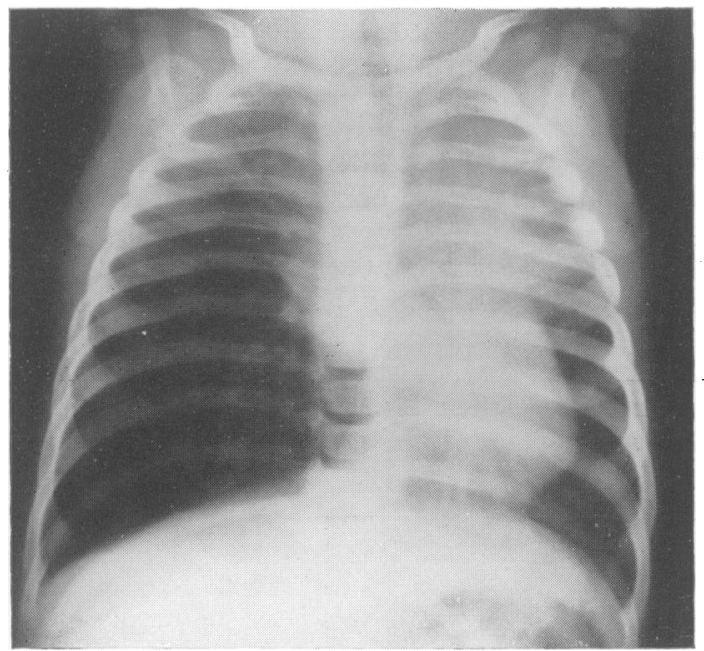

X-RAY 3a.-V.C., girl, 8 months old, October 5, 1953. Primary tuberculosis. Tension emphysema right side with displacement of trachea and widened carina. Bronchoscopy showed right and left main bronchi much narrowed; no ulceration seen.

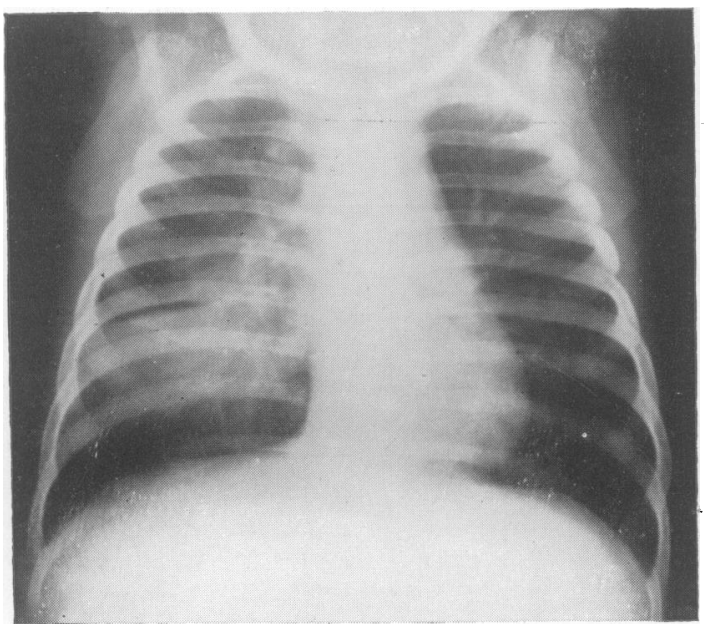

X-RAY 3c.-Same child, December 2, 1953, three weeks later showing re-expansion of right lower lobe and collapse of right middle lobe. infants and young children. These glands owing to their anatomical relation to the bronchi, may $\frac{2}{3}$ ulcerate through the bronchial wall, or bulge the $\underset{\mathbb{Q}}{\stackrel{2}{ }}$ bronchial wall, altering the size of the lumen of one $c$ of the larger bronchi. If the lumen is occluded,

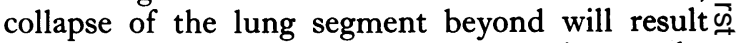
and the X-ray will show an opacity due to the collapsed lobe or segment. Incomplete obstruction may give rise to tension emphysema of the $\frac{\bar{\omega}}{\partial}$ affected lobe. Collapse of one lobe or segment $\stackrel{\Phi}{\Phi}$ may be found together with tension emphysema in another part of the lung. (X-ray $3 \mathrm{a}, \mathrm{b}, \mathrm{c} \& \mathrm{~d}$ ).

$\mathrm{X}$-rays may show a homogenous shadow suggest $\cdots$ ing consolidation of a lobe or segment in which $\vec{\omega}$ there may be cavitation. This appearance occurso

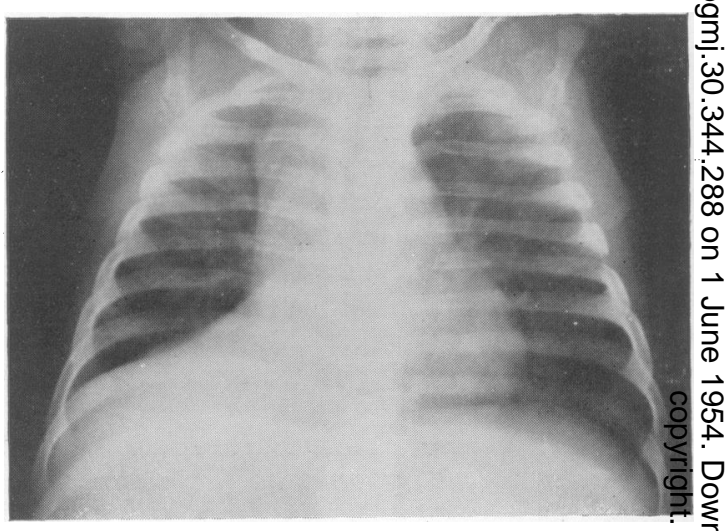

X-RAY 3b.-Same child, November I 3, r 953, five weeks $\overline{\bar{O}}$ later and ten days after bronchoscopy, showing collapse of right lower lobe.

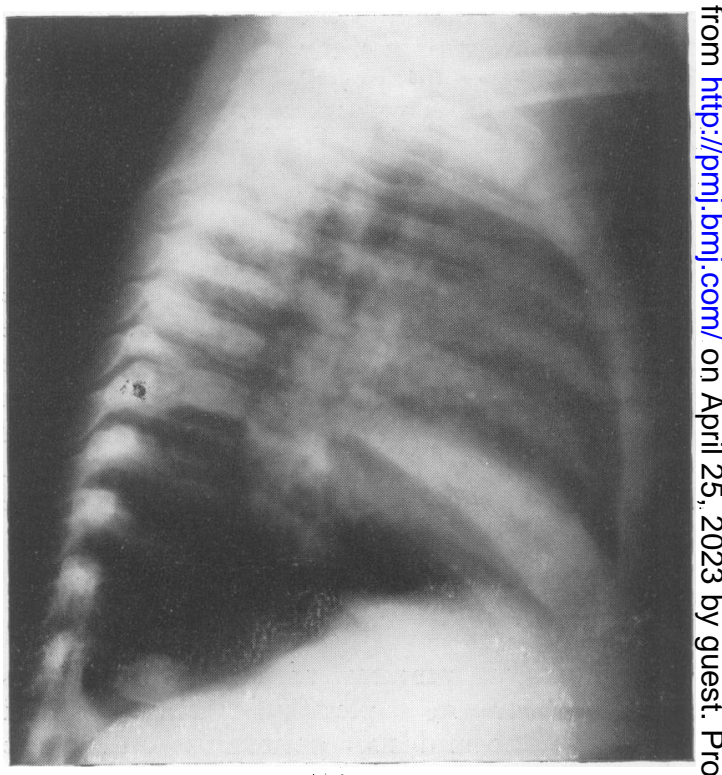

X-RAY 3d.-December 2, i 953. Lateral view, showing collapse of middle lobe. 
in subacute or chronic caseating pneumonia developing either from primary pulmonary lesions or by bronchial spread.

\section{Symptoms and Signs}

Although radiograms may show large hilar shadows, there may be no symptoms and the child may appear to be quite healthy and the X-ray findings come as a surprise. It is not infrequent, however, to find evidence of some ill-health, such as loss of energy, failure to gain weight normally and slight irregular pyrexia.

Involvement of bronchi usually gives rise to localizing symptoms. Cough which is often paroxysmal may develop and a characteristic wheezing is noticed, becoming more marked after exertion or crying. If large sections of lung are involved by collapse or emphysema there may be cyanosis and distressed breathing or reluctance to make any physical effort, even crying, which requires extra exertion. Babies who have not been weaned may not be able to suck properly.

Abnormal physical signs due to primary pulmonary lesions are only evident when there is acute broncho-pneumonia and miliary tuberculosis, collapse, emphysema or caseating pneumonia involving large areas of lung. The involvement of the bronchial wall due to ulceration or pressure from tuberculous glands may give rise to persistent rhonchi in the corresponding bronchus or a persistent inspiratory wheeze.

Collapse, involving large segments or even a complete lobe of a lung may give rise to few abnormal signs. Emphysema of the other portions of lung and possibly fixation of mediastinum due to adenitis may mask the presence of collapse.

Tension emphysema may be detected by fulness of the affected side, displacement of the apex beat and hyper-resonance with absent breath sounds.

Large areas of caseating pneumonia which may be associated with areas of collapse give rise to signs of consolidation and the discrepancy between the signs of consolidation and the general condition of the child, with little malaise and often only slight pyrexia is remarkable and often gives the first clue as to the type of lesion present in the lung.

\section{Management and Treatment of Primary Tuberculosis in Children}

For acute tuberculosis in childhood, including acute bronchopneumonia, miliary tuberculosis and meningitis, treatment usually follows orthodox lines. Streptomycin, para-amino salicylic acid and isoniazide are used in varying combinations with the addition of tuberculin in meningitis, the results of treatment varying more consistently with the stage of the disease at which treatment is begun than with the variations in technique or treatment. Details of such treatment need not be discussed here.

The management of primary tuberculous lesions with less marked constitutional disturbance or with none at all, gives rise to a greater degree of difference of opinion.

It is, however, clear that the risks involved as a result of contact with open cases of tuberculosis in early childhood and probably also in adolescence, are so serious that measures should be taken to prevent heavy infection, especially at these ages.

\section{Prevention}

Bovine infection is preventable and in large towns is probably of insignificant account as a source of tuberculous disease. By adequate pasteurisation of milk and by the use of milk from tuberculin tested herds infection due to bovine tuberculosis disappears. In country districts where unpasteurized milk is provided from non-attested herds, the risk of infection can be reduced or abolished by boiling milk before use.

Infection from human sources is more difficult to control. As soon as a source of active tuberculosis is discovered, any young child in the household or in close proximity should be kept away until such time as the tuberculous patient is removed elsewhere. Isolation of the child from the possible source of infection may be impossible owing to inco-operation of parents, or, unfortunately, to lack of hospital accommodation and to poor housing.

A considerable number of children are infected by the time diagnosis of tuberculosis is made in the ' contact' case: this fact stresses the importance of detecting active tuberculosis as early as possible and justifies regular radiographic examination as well as early investigation of any symptom which may be suspicious of tuberculosis in persons who come into contact with young children.

In late childhood and adolescence it is less easy to control contact with infection where the source of infection is often undetectable.

\section{B.C.G. Inoculation}

B.C.G. inoculation is indicated when it is impossible to remove the child from infection and when there is a possible risk of infection due to reactivity of a quiescent case in the child's environment. It is also desirable in late childhood and adolescence when the possibility of infection increases with a widening of the young person's horizon. By providing the child with a degree of immunity, the inoculation guards it from undesirable complications of primary infection of the lungs or intestine due to a heavy and uncontrolled first infection. It should however be impressed on 
parents that this immunity is not complete and does not obviate care in the prevention of frequent and heavy infection, nor is the immunity permanent. Repetition at yearly intervals of tuberculin tests should be carried out to ensure that the B.C.G. inoculation is still effective. In older children, small exogenous reinfections frequently maintain hypersensitivity to tuberculin tests, but at this age also heavy and repeated infections should be avoided. When there is known risk of infection, special care must still be taken in supervising such inoculated children and adolescents by regular radiographic examination.

\section{Treatment of Primary Tuberculous Lesions}

Many children with primary lesions do not require treatment. An analysis of 224 contact children between ages of birth to 15 years who attended the contact clinic at Brompton Hospital during the years 1948 to 195 I gives some indication of the number who were considered to need some form of treatment or special care. These children were all known to have been in close contact with someone with positive sputum or with evidence of a tuberculous cavity; the group contains only those who were positive to tuberculin tests when first examined.

Of these 224 children, II 7 (52.8 per cent.) had no radiographic evidence of primary lesions; $4 \mathrm{I}$ (18.7 per cent.) had evidence of calcification only, and 63 (28.5 per cent.) had signs which were interpreted as being due to active tuberculous lesions. The $4 \mathrm{I}$ children with calcified lesions had not had any treatment and therefore they had dealt with their primary lesions satisfactorily without treatment. The 117 with no radiographic evidence of primary lesions were followed for a minimum of one year and developed no evidence during that time of further disease, therefore it may fairly be presumed that there was no indication for treatment in this group. Sixty-three (28.5 per cent.) children had evidence of active tuberculous lesions, but in only 49 (21.9 per cent.) of these was the lesion such that it was considered to require special care.

Brian Thcmpson gives figures for children under 14 years attending Ealing Club Clinic during the years 1942 to $1945 .^{4}$ There were 604 children with positive tuberculin tests on first examination and $5 \mathrm{I}$ who converted from negative to positive tests. Among these 655 infected children there were I 30 (I9 per cent.) with lesions " which would reasonably be regarded as part of a primary infection' on first examination or on conversion, a figure which corresponds closely to that of 21.9 per cent. quoted above. These figures give an indication of the significance which should be attached to tuberculous infection during child hood as a whole.

From what has already been said, however, it iक्क़ obvious that the significance of tuberculous infec $\frac{\varrho}{2}$ tion is closely linked with the age at which it takes. place.

Whether or not treatment is indicated will have to be decided in each case which presents itself according to its circumstances as well as to its age Consideration will have to be given to degree and frequency of infection, social and economige conditions, and also to the character and extent of the lesion found.

The possible existence and the extent of the lesion should not be gauged by one X-ray examina tion. The radiogram may have been taken soong after infection had taken place and before the lesion had fully developed, so that it is advisablè. to make a second X-ray examination within tw\& or three months of the first.

When the only evidence of primary tuberculosis is a moderate degree of hilar adenitis, regula supervision with repetition of radiograms at regulag intervals is all that is indicated as a rule. These children are very unlikely to be a source of infection and need not be segregated from othe children. When of school age they can usually continue to attend school.

Gross hilar enlargement and any complication $+{ }_{s}^{+}$ indicating bronchial involvement will requipire more definite treatment. Opinions at the presens time are not unanimous concerning the value of rest in bed for these lesions. The experience o\% wise clinicians in the days before antibiotics an surgical treatment were available should not, think, be lightly cast aside and they were fully aware of the value of rest in healing active tuberculous lesions. Insistence on rest in bed i the early stage of large lesions, if necessary for period of months, may prevent further compliz. cations and may result in complete recovery os lesions which at first were extensive. A perio of rest in bed of four or six months in congenia surroundings is no great hardship for a young child, and is worth while if the child is saved even 9 minimal risk of serious complications.

Whether rest is provided at home or in hospit重. or sanatorium will depend largely on hom\& circumstances. Apart from necessary room, fresh air and good food a child must have company an entertainment or employment if it is going to stay in bed at all.

In the more acute cases and in those with stridor, it is advisable to have the child undeen supervision and in expert care. Early signs of complications such as meningitis or bone lesion\$ are usually more readily detected when the chil 8 is in an institution. 


\section{Streptomycin, para-amino salicylic acid and isoniazide}

In the acute forms of tuberculosis antibiotics are of great value. It is doubtful whether they have any definite use in the treatment of large caseating glands or chronic caseating pneumonia. Neither the size of the caseating lesions nor the rate of healing is dramatically influenced. There is not at present sufficient evidence to assess their value in those cases where ulceration of a bronchus is present.

Since it is probable that haematogenous infection to a greater or less degree arises in many primary lesions, especially in infants, antibiotics are given by some physicians to young children who have evidence of recent infection with definite primary lesions, hoping in this way to reduce the possibility of miliary lesions both evident and occult.

\section{Bronchoscopy and Surgical Treatment}

Treatment when a bronchus has become involved in the primary lesion presents many problems. It may be necessary, though rarely, as an emergency measure to clear the lumen of a bronchus of tuberculous material when stridor is marked and endangering a child's life. Bronchoscopic removal of the material may be attempted or, alternatively, thoracotomy and removal of the mass of glands invading the bronchial wall. Bronchoscopy may be indicated for removal by suction of pus from bronchi, beyond the partial obstruction, which have become secondarily infected. Richards and Blair ${ }^{6}$ recommend routine bronchoscopy in order to reduce the possibility of bronchiectasis developing in the collapsed lobe.

How to treat the collapsed lobe is still a debatable point. That bronchiectasis may result from such collapse is an established fact. As yet the number of cases with collapse which develop bronchiectasis with symptoms in later life is not known. Prevention and treatment of secondary infection in the collapsed segment is no doubt of importance. The problem of the collapsed lobe is at present being dealt with usually in one of three ways. Expectant treatment is advocated by some on the assumption that the majority heal sufficiently to allow complete or partial re-expansion of the collapsed lobe and, in only a few cases, will bronchiectasis develop later; others recommend resection of the collapsed lobe, or segment, either at an early stage or after a period of a year or more if there has not been complete recovery, so preventing future trouble in an unexpanded or partially re-expanded segment. The third form of treatment is thoracotomy and removal of the mass of caseating glands in order to prevent possible irreversible collapse and development of bronchiectasis.

The treatment of chronic caseating lesions occupying large areas of the lung is also varied. With our present knowledge of this type of lesion it is not possible to say how many of them heal completely, although apparently there are some which do so. It is clear that many of them remain in a chronic state for a year or more. It has been suggested that parts of these lesions may remain unhealed and, in later life, give rise to clinical pulmonary tuberculosis. On this assumption, it is suggested that removal of such caseating lung lesions is preferable to a 'wait and see' policy.? On the one hand there is the risk of the operation and the possible disadvantage to a child of the loss of a lobe or even a complete lung and, on the other, the disadvantage of a long period of treatment probably away from home and the possible relationship of these lesions to clinical tuberculosis in later life.

\section{REFERENCES}

1. MEDICAL RESEARCH COUNCIL, National Tuberculin Survey (1952), Lancet, i, 775 .

2. HYGE, T. V. (1945), Ugesk. f. lacger, 105, 779

3. CAMMOCK, R. M., and MILLER, F. J. W. (1953), Lancet, i, 158.

4. BEAVEN, P. W. (1950), Dis. of Chest, 17, 280.

5. THOMPSON, B. C. (1952), Proc. Roy. Soc. Med., 45, 741 .

7. THOMAS, D. (1952), Proc. Roy. Soc. Med., 45, 743.

Continuation of Bibliography-Fohn C. Roberts, M.D., M.R.C.P., from page 287.

\section{BIBLIOGRAPHY}

MAHER-LOUGHNAN, G. P. (1952), Tubercle, 33, 342.

BIGNALL, J. R., CROFTON, J. W., and THÖMAS, J. A. B. (1951), Brit. med. F, i, 554 . Admin. Conf. on Chemotherapy of Tuberculosis.

SUMNER, J. (1949), Tubercle, 3, 62.

CROFTON, J. (1952), Brit. med. $\mathscr{F}$., ii, 1014.

ANNOTATION (1953), Lancet, i, $23 \mathrm{I}$. JONES, E.' R. (1952), Tubercle, 33, 369 .

HOUGHTON, L. E. (1952), Ibid., 33, 381.

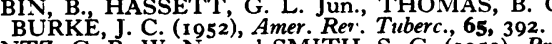

LUNTZ, G.' R. W. N., and SMITH, S. G. (1953), Brit. med. F.,

ROSS-COSBIE, J., GOW, J. G., and ST. HILL, C. A. (1953),

Ibid.ji, 90I.
TURNBULL, F. W. A., WALLACE, A. T., STEWART, SHEILA, and CROFTON, J. W. (I953), Ibid., i, I 244

MCCARTHY, D., and MANN, T. P. (1950), Ibid., i, 34 I.
BARNETT, MARGARET, BUSHBY, S. R. M., and MITCHESÓN, D. A. (I953), Lancet, i, 314.
JOINER, C. L., MACLEAN, K. S.,PRITCHARD, E. K., ANDER-
SON, K., COLLARD, P., KING, M. B., and KNOX, R. (rON2, Kbid., ii, 843.

MEDICAL RESEARCH COUNCIL REPORT No. 4 (1953), Ibid., ii, 217 .

LORBER, J. (1950), Ibid., i, 389.

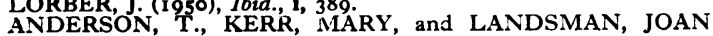
(I953), Ibid., ii, 691 .

RITCHIE, M., 'TAYLOR, R. M., and DICK, J. C. (1953), Ibid., ii, 419.

TORRES-GOST, J. (1953), Ibid., ii, 693

FLETCHER, A. P. (1953), Ibid., ii, 694 .

CA'THIE, L. A. P. (1950), Ibid., i, 441 .

SMITH, HONOR V., and VOLLƯM, R. L. (1950), Ibid., ii, 275.

EVANS, E. T. (1952), Ұ. Bone and Foint Surg., 2, 267.

DEROY, M. S., and FISHER, H. (1952), Ibid., 2, 299.

DICK, J. C. (1953), Lancet, i, 808.

BURNS, T., SMITH, H. G. M., and SNAITH, L. M. (1953), Ibid., i, 81 7 . 